\section{Inside the 2015 ASCO Genitourinary Cancers Symposium}

\author{
Matteo Santoni ${ }^{*}, 1$, Francesco Massari ${ }^{2}$, Roberto lacovelli ${ }^{3}$, Chiara Ciccarese ${ }^{2}$, \\ Elena Verri ${ }^{3}$, Luciano Burattini', Rodolfo Montironi ${ }^{4}$, Franco Nolè ${ }^{3}$, \\ Giampaolo Tortora² \& Stefano Cascinu ${ }^{1}$
}

\section{The American Society of Clinical Oncology (ASCO) Genitourinary Cancers Symposium, Rosen Shingle Creek, Orlando, FL, USA, 26-28 February 2015}

The American Society of Clinical Oncology (ASCO) Genitourinary Cancers Symposium was held in Orlando (FL, USA), from 26 to 28 February 2015. This meeting was focused on 'Integrating Biology into patient-centric care' and represented an attractive opportunity for oncology professionals with a special interest in the diagnosis and treatment of genitourinary tumors. The identification and validation of biomarkers for tumor response had been the focus of several researchers at the symposium, together with the development of novel targeted agents. This report is a summary of the highlights on kidney and prostate tumors presented at the 2015 ASCO Genitourinary Cancers Symposium by various investigators.

The 2015 ASCO Genitourinary Cancers Symposium has been a unique opportunity to show the emerging results focused on genitourinary malignancies, debate the impact of recently validated evidences on daily clinical practice and suggest the future research directions in this field.

Currently, there are two main areas of research. RCC issues include the identification of the molecular background of RCC pathogenesis and progression, and the validation of predictive and prognostic factors. The second research field concerns the optimization of radiation therapy for patients with localized prostate cancer and the identification of novel targeted agents for the treatment of metastatic castration resistant prostate cancer (mCRPC). In the following sections, we discuss the results of selected studies focusing on these areas.

\section{Renal cell carcinoma: the failure of adjuvant therapy $\&$ the research of prognostic factors \\ During the meeting, Dr Haas presented the initial results of the ASSURE (E2805) study, a large multicenter randomized Phase III trial that is assessing the efficacy of two oral multitargeted VEGF inhibitors, sunitinib and sorafenib, in the adjuvant setting [1]. The study included 1943 patients with completely resected RCC at high risk of recurrence. Patients were randomized to 1 year of treatment with sorafenib (400 mg twice daily), sunitinib $(50 \mathrm{mg} /$ day for 4 of every 6 weeks) or placebo. The results fell far short of the $25 \%$ reduction mark at an interim analysis conducted with $62 \%$ of the anticipated data. Using a stratified log-rank test, 5-year disease-free survival (DFS) rates varied little across the three arms at 52.8-55.8\%, yielding hazard ratios (HRs) of 0.98 for sorafenib and 1.01 for \\ 'Department of Medical Oncology, AOU Ospedali Riuniti - Polytechnic University of the Marche Region, Ancona, Italy \\ ${ }^{2}$ Department of Medical Oncology, University of Verona, Verona, Italy \\ ${ }^{3}$ Medical Oncology Unit of Urogenital \& Head \& Neck Tumors, European Institute of Oncology, Via Ripamonti 435, 20141, Milan, Italy \\ ${ }^{4}$ Pathological Anatomy, Polytechnic University of the Marche Region School of Medicine United Hospitals, Via Conca 71 I-60126, \\ Torrette, Ancona, Italy \\ *Author for correspondence: Tel.: +39 071596 4263; Fax: +39 071596 4269; mattymo@alice.it}

\section{KEYWORDS}

- biomarkers • prostate cancer $\bullet$ renal cell carcinoma

- targeted therapy

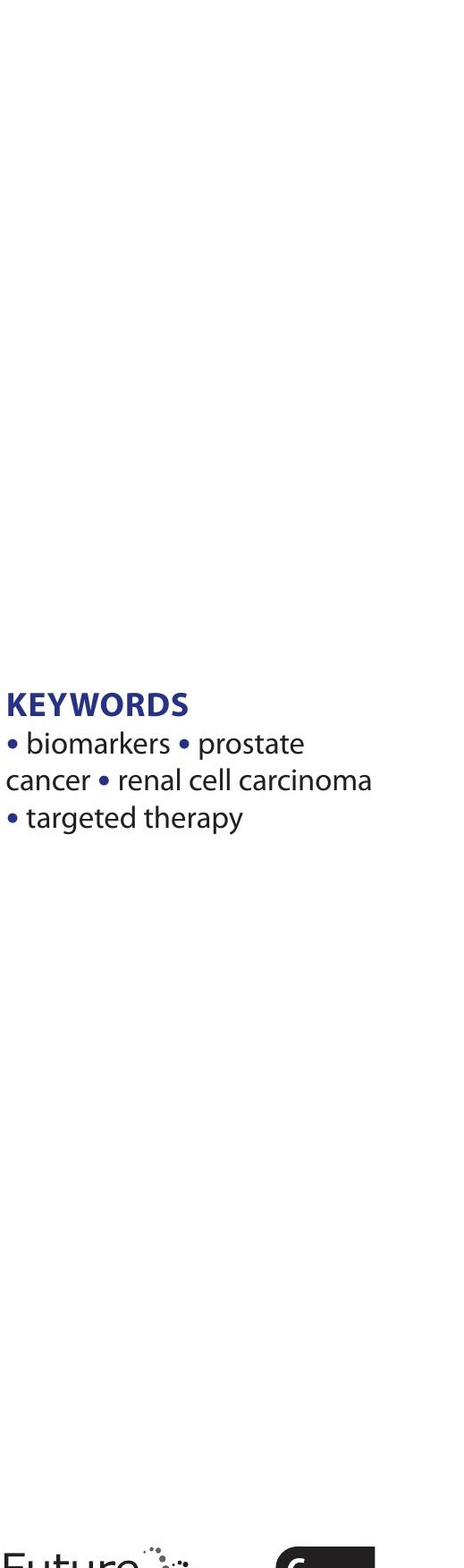


sunitinib in comparison with placebo. Similarly, 5 -year overall survival (OS) ranged little, from a low of $76.9 \%$ to a high of $80.7 \%$, yielding HRs of 0.93 for sorafenib and 1.10 for sunitinib in comparison with placebo. DFS reached 5.6 years with either sorafenib or sunitinib, as compared with 5.7 years with placebo, thus indicating that neither sorafenib nor sunitinib offer benefits in this setting.

Another very attractive study was presented by Dr Alimohamed concerning the prognostic and predictive role of neutrophil to lymphocyte ratio (NLR) [2]. NLR has been shown to correlate with the outcome of patients who underwent nephrectomy for localized RCC, as well as with the response to sunitinib and everolimus [3-7]. At this Congress, Dr Alimohamed and his group evaluated whether NLR is independently prognostic when adjusted for the International mRCC Database Consortium (IMDC) model and evaluated change in NLR ('NLR conversion') as a predictive marker of response to targeted therapy. In this study, NLR $>3.0$ at baseline was independently associated with OS and PFS in both the training and validation cohorts. A decrease in NLR by week 6 was associated with longer OS (21.1 vs 9.7 months; HR: 0.57; $\mathrm{p}<0.001$ ), PFS (8.8 vs 4.6 months; HR: 0.54; $\mathrm{p}<0.001$ ), and higher objective response rates (35 vs $13 \%$; $p<0.001$ ) compared with patients without a decrease. Therefore NLR would seem an independent prognostic factor after adjusted for IMDC criteria.

In 2014, Dr Albiges et al. reported that higher BMI $\geq 25 \mathrm{~kg} / \mathrm{m}^{2}$ was associated with better OS (median: 25.6 vs 17.1 months; HR: $0.84 ; \mathrm{p}=0.0079)$ and longer time to treatment failure (median: 8.1 vs 5.7 months; HR: 0.86; $\mathrm{p}=0.0067)$ in 1975 patients with mRCC from clinical trials [8].

At the present meeting, the authors conducted an external validation study using data from 4657 patients with mRCC treated in Phase II and III clinical trials from 2003 through 2013 [9]. They confirmed that BMI $\geq 25$ was associated with better outcomes in terms of OS (23.4 vs 14.5 months; $\mathrm{p}=0.0008)$, progression-free survival (8.2 vs 5.5 months; $\mathrm{p}<0.0001)$ and overall response rate ( 25.3 vs $17.6 \%$; $p<0.0001)$. Furthermore, the investigators integrated data from The Cancer Genome Atlas (TCGA) dataset on patients with metastatic clear cell RCC (ccRCC) to confirm the prognostic role of BMI and to see whether there was correlation between BMI and the expression of FASN. Although they did not detect a significant survival advantage for higher BMI in this cohort, they found that higher BMI was significantly associated with lower FASN expression levels $(\mathrm{p}<0.034)$, and that FASN expression below the median level was associated with better OS $(p<0.002)$. Finally, the authors found that FASN staining was associated with prognostic groups, but was not an independent prognostic factor for OS in multivariable analysis.

As for the proteomic stratification of ccRCC, Dr Kaffenberg described reversed-phase protein array clustering of 324 patients from the ccRCC TCGA that revealed five different clusters characterized by alterations in specific pathways [10] . Cluster 1 was correlated with poor diseasespecific survival (DSS), decreased expression of RTK and upregulation of the mTOR pathway. It was also associated with mTOR pathway genomic alterations, sarcomatoid histology and the $c c B$ prognostic mRNA signature $(\mathrm{p}<0.001)$. Cluster 2 was characterized by increased expression of RTKs and an important upregulation of the mTOR pathway with good DSS. Cluster designation persisted independently associated with DSS for stage and grade (HR: 0.23 for cluster 2; 95\% CI: $0.08-0.68 ; \mathrm{p}=0.008)$.

Therefore, after the external validation, the authors identified two distinct mTOR-activated clusters (one with high RTK activity and one with increased mTOR pathway genomic alterations), which may have prognostic and therapeutic future implications.

\section{Prostate cancer: radiotherapy \& new tyrosine kinase inhibitors}

Two large Phase III trials in patients affected by prostatic cancer have been presented at this meeting, one about organ confined disease and another in pretreated mCRPC. The first one investigated if the dose escalation up to $79.2 \mathrm{~Gy}$ with $3 \mathrm{D}$ conformal radiotherapy (3DCRT) or with the intensity-modulated radiation therapy (IMRT) will improve OS compared with the 70.2 Gy 3DCRT or IMRT in patients affected by localized prostate cancer [11]. The inclusion criteria of the study were stage cT $1 b-\mathrm{T} 2 \mathrm{~b}$ with Gleason score 2-6 and PSA $\geq 10$ and $<20$ or GS 7 and PSA $<15$. The primary end point was to reach a decrease of the risk of death of $23 \%$ with an expected HR of 0.77 in favor of $79.2 \mathrm{~Gy}$. Secondary end points were biochemical failure, time to local progression, distant metastases, prostate cancer-specific survival and late 
gastrointestinal or genitourinary toxicities. A total of 1532 patients were randomized 1:1, the median age was 69 years and $70 \%$ of patients had PSA $<10 \mathrm{ng} / \mathrm{ml}, 84 \%$ had Gleason score 7 , $57 \%$ had $\mathrm{T} 1$ disease and 66\% used 3D-CRT. After a median follow up of 7 years, the 5-year OS rate was 88 and $89 \%$ and the 10 -year OS rate was 67 and $66 \%$ for 79.2 and $70.2 \mathrm{~Gy}$, respectively (HR: $0.98 ; 95 \%$ CI: $0.79-1.21 ; \mathrm{p}=0.87$ ). Based on this result, the study was considered negative even if the 79.2 Gy 3DCRT/IMRT was able to improve the 5- and 10-year rate of biochemical failure $(\mathrm{p}<0.001)$, the local progression (HR: 0.46 ; 95\% CI: $0.27-0.81 ; \mathrm{p}=0.006$ ), the onset of distant metastases (HR: 0.57; 95\% CI: $0.35-0.94 ; \mathrm{p}=0.003)$ and decreased the rate of salvage therapy $(13.5$ vs $21 \% ; \mathrm{p}=0.0002)$. All together, these results were consistent with previous experiences that failed to report an improvement of OS with increased dose of radiation therapy for localized prostate cancer.

The second trial investigated the activity of cabozantinib, a small molecule receptor tyrosine kinase inhibitor, in mCRPC patients [12]. Cabozantinib strongly binds to and inhibits several TKs such MET, RET, VEGFR1-3, KIT and others. Cabozantinib reported a promising activity in a Phase II trial in mCRPC and results of the Phase III randomized, doubleblind COMET-1 trials were presented at this meeting. Main inclusion criteria for patient enrollment were: diagnosis of CRPC, previous treatment with docetaxel and with abiraterone and/or enzalutamide. The primary end point of the study was OS with an expected HR of 0.75 . A total of 1028 patients were randomized 2:1 to receive cabozantinib at $60 \mathrm{mg} /$ day or prednisone $5 \mathrm{mg} /$ day. The final result reported no significant differences in OS (11.0 vs 9.8 months), with an HR of 0.90 (95\% CI: 0.76-1.06; $\mathrm{p}=0.213$ ). About secondary end points, the bone scan response at 12 weeks was greater in patients treated with cabozantinib ( 42 vs $3 \%$; $\mathrm{p}<0.001$ ), as well as the PFS (5.6 vs 2.8 months; $\mathrm{p}<0.001$ ), and the time to the first skeletal event (HR: 0.62; 95\% CI: 0.78-0.81; $\mathrm{p}<0.001)$. However, the higher rate of adverse events with cabozantinib (33 vs $12 \%$ ) and the high drug dosage compared with previous studies might have limited patient compliance and affected the final results.

\section{Conclusion}

The 2015 Genitourinary Cancers Symposium has been the mirror of the changing landscape in renal and prostate malignancies. Promising predictive and prognostic biomarkers were presented for patients with renal and prostate tumors, supporting their employment in directing clinician's therapeutic strategies and in the design of clinical trials. However, a better knowledge of the molecular mechanisms underlying tumor progression and primary/acquired resistance to therapy is dramatically needed in order to develop effective therapeutic approaches for patients with localized or metastatic genitourinary tumors.

\section{Financial \& competing interests disclosure}

The authors have no relevant affiliations or financial involvement with any organization or entity with a financial interest in or financial conflict with the subject matter or materials discussed in the manuscript. This includes employment, consultancies, honoraria, stock ownership or options, expert testimony, grants or patents received or pending, or royalties.

No writing assistance was utilized in the production of this manuscript.

\section{References}

1 Haas NB, Manola J, Uzzo RG et al. Initial results from ASSURE (E2805): adjuvant sorafenib or sunitinib for unfavorable renal carcinoma, an ECOG-ACRIN-led, NCTN Phase III trial. J. Clin. Oncol. 33(Suppl. 7), Abstract 403 (2015).

2 Alimohamed NS, Templeton AJ, Knox JJ et al. Change in neutrophil to lymphocyte ratio as a prognostic and predictive marker in response to targeted therapy for metastatic renal cell carcinoma. J. Clin. Oncol. 33(Suppl. 7), Abstract 404 (2015).

3 Ohno Y, Nakashima J, Ohori M, Gondo T, Hatano T, Tachibana M. Follow up of neutrophil-to-lymphocyte ratio and recurrence of clear cell renal cell carcinoma. J. Urol. 187, 411-417 (2012).

4 Ohno Y, Nakashima J, Ohori M, Hatano T, Tachibana M. Pretreatment neutrophil-tolymphocyte ratio as an independent predictor of recurrence in patients with non-metastatic renal cell carcinoma. J. Urol. 184, 873-878 (2010).

5 Santoni M, Buti S, Conti A et al. Prognostic significance of host immune status in patients with late relapsing renal cell carcinoma treated with targeted therapy. Target Oncol. (2015) (In Press).

6 Keizman D, Ish-Shalom M, Huang P et al. The association of pre-treatment neutrophil to lymphocyte ratio with response rate, progression free survival and overall survival of patients treated with sunitinib for metastatic renal cell carcinoma. Eur. J. Cancer 48, 202-208 (2012).

7 Santoni M, De Giorgi U, Iacovelli R et al. Pre-treatment neutrophil-to-lymphocyte ratio may be associated with the outcome in patients treated with everolimus for metastatic renal cell carcinoma. Br. J. Cancer 109, 1755-1759 (2013).

8 Albiges L, Xie W, Lee JL et al. The impact of body mass index (BMI) on treatment outcome of targeted therapy in metastatic renal cell carcinoma (mRCC): results from the International Metastatic Renal Cell Cancer Database Consortium. J. Clin. Oncol. 32(Suppl. 5), Abstract 4576 (2014). 
9 Albiges L, Hakimi AA, Lin X et al. The impact of BMI on outcomes of patients with metastatic renal cell carcinoma treated with targeted therapy: an external validation data set and analysis of underlying biology from The Cancer Genome Atlas. J. Clin. Oncol. 33(Suppl. 7) Abstract 405 (2015).

10 Kaffenberger SD, Ciriello G, Winer AG et al. Proteomic stratification of clear cell renal cell carcinoma utilizing The Cancer Genome Atlas (TCGA) with external validation. J. Clin. Oncol. 33(Suppl. 7), Abstract 406 (2015).

11 Michalski JM, Moughan J, Purdy J et al. A randomized trial of 79.2Gy versus 70.2Gy radiation therapy (RT) for localized prostate cancer. J. Clin. Oncol. 33 (Suppl. 7), Abstract 4 (2015).
12 Smith MR, De Bono JS, Sternberg CN et al. Final analysis of COMET-1: Cabozantinib (Cabo) versus prednisone (Pred) in metastatic castration-resistant prostate cancer (mCRPC) patients (pts) previously treated with docetaxel (D) and abiraterone (A) and/or enzalutamide (E). J. Clin. Oncol. 33(Suppl. 7), Abstract 139 (2015). 